\title{
On the relation between ionospheric parameters and sunspot number ${ }^{\dagger}$
}

\author{
Chris Hall ${ }^{*}$ (D) and Magnar Gullikstad Johnsen \\ Tromsø Geophysical Observatory, UiT - The Arctic University of Norway, 9037 Tromsø, Norway \\ *Corresponding author. Email: chris.hall@uit.no
}

(Received 27 March 2020; Accepted 20 April 2020)

\begin{abstract}
In a recent study, mid-latitude ionospheric parameters were compared with solar activity; it was suggested that the relationship between these, earlier assumed stable, might be changing with time (Lastovicka, 2019). Here, the information is extended to higher latitude $\left(69.6^{\circ} \mathrm{N}, 19.2 \mathrm{E}\right)$ and further back in time. For the ionospheric F-region (viz. the critical frequency, FoF2) the same behaviour is seen with a change-point around 1996. For the ionospheric E-region (viz. the critical frequency, foE), change-points are less obvious than in the mid-latitude study, presumably owing to the observation site lying under the auroral oval.
\end{abstract}

Keywords: ionospheric critical frequencies; sunspot-number; trends

\section{Introduction}

With an increasingly long time-series of parameters from the upper atmosphere, it is becoming viable to investigate climatology. Upper atmosphere trends or change can reflect corresponding ones in the troposphere (Hall et al., 2007; Roble \& Dickinson, 1989), but at an altitude not directly influenced by local short-term effects. One endeavours to establish the mechanisms responsible for observed trends, one contender being solar forcing: the focus of this study and that of Lastovicka (2019). Once this effect has been removed, it may be possible to identify others, including anthropogenic causes, if any.

\section{Objective}

The results of Lastovicka (2019) do not include observations from the auroral region nor from earlier than 1980. The observations in Troms $ø\left(69.6^{\circ} \mathrm{N}, 19.2 \mathrm{E}\right)$ going back to the 1930 s provide these additions, thus supplementing Lastovicka's study.

\section{Methods}

The parameters used here are obtained from the ionosonde located near Tromsø, Norway $\left(69.6^{\circ} \mathrm{N}\right.$, $19.2 \mathrm{E}$ ) and in particular the local noon critical frequencies of the ionospheric E- and F2-layers ("foE" and "foF2" respectively, in MHz). The instrument is fully described by Hall and Hansen (2003). The underlying physics of the ionosphere is reliably described by Ratcliffe (1972). The principles of ionosonde

\footnotetext{
${ }^{\dagger}$ The online version of this article has been updated since original publication. A notice detailing the changes has been published. 

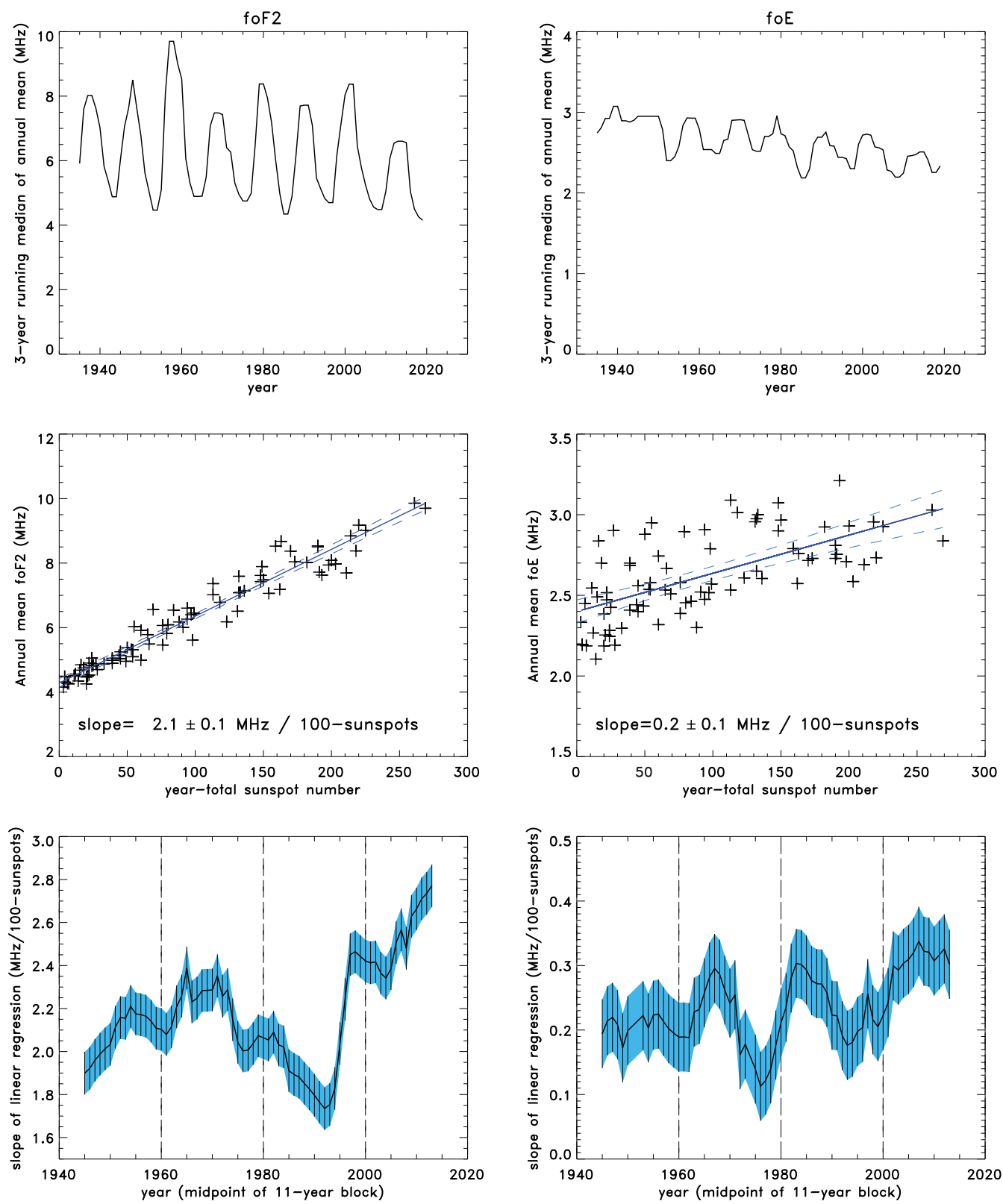

Figure 1. Underlying data and subsequent analyses of the foF2 and foE time series. Left-hand column: foF2; right-hand column: foE. Top row: annual means of local noon observations, and with a 3-year running median filter to remove anomalous years (e.g. featuring instrument-changes etc.). Centre row: regressions on yearly total sunspot numbers obtained from WDC SILSO, Royal Observatory of Belgium; dashed lines show the $95 \%$ confidence limits and annotation shows the slopes of the regression and 1- $\sigma$ uncertainty. Bottom row: 11-year running regression on corresponding sunspot number, replicating the approach of Lastovicka (2019); vertical bars and shading indicate $1-\sigma$ uncertainties.

operation are fully accessible in Reinisch (1996) and references therein. The international standard reference for scaling ionograms in order to determine foE and foF2 is the URSI handbook of ionogram scaling and reduction available here:

http://www.sws.bom.gov.au/IPSHosted/INAG/uag_23a/uag_23a.html (accessed 2020.02.28). 
Corresponding sunspot numbers as yearly totals were obtained from the World Data Center SILSO, Royal Observatory of Belgium, Brussels. Lastovicka (2019) used F10.7 rather than the sunspot-number. However, the dataset presented here goes further back in time than the available F10.7, hence the choice of sunspot number.

Linear regressions were performed on running 11-year periods for foE and foF 2 and corresponding periods of sunspot numbers (identical to the method used by Lastovicka).

\section{Results}

In Figure 1, the results are grouped as two parameters, foE $(\mathrm{MHz})$ and foF2 $(\mathrm{MHz})$. For each, the annual means are portrayed and with a 3-year running median applied to remove misleading spikes. In both cases the 11 year periodicity of the solar cycle is very evident (sunspot number is not shown explicitly in here as it is readily available elsewhere). The time series show results from the start of regular operation in 1938. For each parameter, the solar dependence is demonstrated by a linear regression including the $95 \%$ confidence limits (Working \& Hotelling, 1929): $2.1 \pm 0.1 \mathrm{MHz} / 100$ sunspots, for foF 2 and a high degree of confidence; $0.2 \pm 0.1 \mathrm{MHz} / 100$ sunspots, for foE and a considerably lower degree of confidence. The latter, foE, demonstrates the much weaker dependence compared with foF2 as shown by Lastovicka (2019), but considerably more variable, reflecting the mechanisms in the auroral zone. A caveat here is that sunspot-number can be used as a proxy for f10.7 flux, although Lastovicka (2019) suggests this may not be as well defined as hitherto assumed.

\section{Discussions}

The existence, of the F2 layer is due to a combination of electron production, loss and advection, not necessarily in that order. The E-region, on the other hand is characterised as a typical Chapman phenomenon with ionisation and recombination occupying shorter timescales (e.g. Hargreaves, 1992). Solar forcing is responsible for initial ionisation, but thereafter observations of the F2-layer do not necessarily reflect that forcing of the local ionosphere; on the other hand, presence of the E-region is the result of solar origins above the geographic location of the observation. It is not surprising, therefore that the respective responses of foF 2 and foE to sunspot number are very different. Compounding this, E-layer production in the auroral zone is considerably influenced by energetic precipitation originating from enhanced solar activity compared with equatorward latitudes (Qian et al., 2020).

\section{Conclusions}

For the high-latitude F2-layer, there is a distinct increase in dependence of critical frequency on sunspotnumber in 1996, in full agreement with similar observations at mid-latitude (Lastovicka, 2019). A somewhat gradual decrease in dependence can be seen between 1960 and 1995. For the corresponding E-layer, however, the increase reported by Lastovicka (2019) for 2000 is not at all obvious, whereas the dependence since 1960 is more characterised by large fluctuations; we attribute this difference to processes in the auroral region not present at lower latitudes.

Funding Information. This research received no specific grant from any funding agency, commercial or not-for-profit sectors. Conflict of interest. There is no conflict of interest - the contribution is purely supplementary to a publication.

Data availability. Data used in this study are available from Tromsø Geophysical Observatory as part of its national obligation, and as a department of UiT - The Arctic University of Norway - https://en.uit.no/startsida.

\section{References}

Hall, C. M., Brekke, A., \& Cannon, P. S. (2007). Climatic trends in E-region critical frequency and virtual height above Tromsø $\left(70^{\circ} \mathrm{N}, 10^{\circ} \mathrm{E}\right)$. Annals of Geophysics, 25, 2351-2357.

Hall, C. M., \& Hansen, T. L. (2003). 20th century operation of the Tromsø ionosonde. Advances in Polar Upper Atmosphere Research, 17, 155-166.

Hargreaves, J. K. (1992). The solar-terrestrial environment. Cambridge University Press. 
Lastovicka, J. (2019). Is the relation between ionospheric parameters and solar proxies stable?. Geophysical Research Letters, 46, 208-213. https://doi.org/10.1029/2019GL085033.

Qian, L., Wang, W., Burns, A. G., Chamberlin, P. C., \& Solomon, S. C. (2020). Responses of the thermosphere and ionosphere system to concurrent solar flares and geomagnetic storms. Journal of Geophysical Research, 125, e2019JA027431. https:// doi.org/10.1029/2019JA027431.

Ratcliffe, J. A. (1972). An introduction to the ionosphere and magnetosphere. Cambridge University Press.

Reinisch, B. W. (1996). Modern ionosondes. In H.Kohl, R.Rüster, \& K.Schlegel (Eds.) Modern ionospheric science-A collection of articles published on occasion of the Anniversary: “50 Years of Ionospheric Research in Lindau."European Geophysical Society. ISBN3-9804862-1-4.

Roble, R. G., \& Dickinson, R. E. (1989). How will changes in carbon dioxide and methane modify the mean structure of the mesosphere and thermosphere?. Geophysical Research Letters, 16, 1441-1444.

Working, H., \& Hotelling, H. (1929). Application of the theory of error to the interpretation of trends. Journal of the American Statistical Association, 24, 73-85.

Cite this article: Hall C, Johnsen MG (2020). On the relation between ionospheric parameters and sunspot number Experimental Results, 1, e13, 1-6. https://doi.org/10.1017/exp.2020.19 


\section{Peer Reviews}

\section{Reviewing editor: Dr. Ashley Smyth}

University of Florida 3463 Soil and Water Sciences Department 18905 SW 280th St. Homestead Gainesville Florida United States 32611-7011

This article has been accepted because it is deemed to be scientifically sound, has the correct controls, has appropriate methodology and is statistically valid, and met required revisions.

doi:10.1017/exp.2020.19.pr1

\section{Review 1: On the relation between ionospheric parameters and sunspot number}

Reviewer: Dr. Jihad Asad

Palestine Technical University, Physics, Po Box 7, Tulkarm, Palestine, State of

Date of review: 08 April 2020

Published online:

(C) The Author(s) 2020 This is an Open Access article, distributed under the terms of the Creative Commons Attribution licence (http://creativecommons.org/licenses/by/4.0/), which permits unrestricted re-use, distribution, and reproduction in any medium, provided the original work is properly cited.

Conflict of interest statement. I declare that there are no conflict of intrests

Comments to the Author: The paper is well organized It is suitable for Experimental Results Journal I think the introduction is too short

\section{Score Card}

Presentation

3.7

Is the article written in clear and proper English? (30\%)

Is the data presented in the most useful manner? (40\%)

Does the paper cite relevant and related articles appropriately? (30\%)

\section{Context}

3.2

Does the title suitably represent the article? (25\%)

Does the abstract correctly embody the content of the article? (25\%)

Does the introduction give appropriate context? (25\%)

Is the objective of the experiment clearly defined? (25\%)

Analysis

Does the discussion adequately interpret the results presented? (40\%)

Is the conclusion consistent with the results and discussion? (40\%)

Are the limitations of the experiment as well as the contributions of the experiment clearly outlined? (20\%) 


\section{Review 2: On the relation between ionospheric parameters and sunspot number}

Reviewer: Dr. Manuel Malaver De la Fuente $\mathrm{e}^{1,2}$ if

${ }^{1}$ Universidad Marítima del Caribe, Departamento de Ciencias Basicas, Catia la Mar, Venezuela, Bolivarian Republic of, 1162

${ }^{2}$ Bijective Physics Institute, Bijective Physics, Idrija, Slovenia

Date of review: 19 April 2020

Published online:

(C) The Author(s) 2020 This is an Open Access article, distributed under the terms of the Creative Commons Attribution licence (http://creativecommons.org/licenses/by/4.0/), which permits unrestricted re-use, distribution, and reproduction in any medium, provided the original work is properly cited.

Conflict of interest statement. The paper is accept and publishable in Experimental Results

Comments to the Author: The paper is excellent and novelty because present an study about the ionospheric parameters and their relations with solar activity

\section{Score Card}

Presentation

5.0

Is the article written in clear and proper English? (30\%)

Is the data presented in the most useful manner? (40\%)

Does the paper cite relevant and related articles appropriately? (30\%)

Context

5.0

Does the title suitably represent the article? (25\%)

Does the abstract correctly embody the content of the article? (25\%)

Does the introduction give appropriate context? (25\%)

Is the objective of the experiment clearly defined? (25\%)

Analysis

Does the discussion adequately interpret the results presented? (40\%)

Is the conclusion consistent with the results and discussion? (40\%)

Are the limitations of the experiment as well as the contributions of the experiment clearly outlined? $(20 \%)$ 